\title{
A prática desportiva dos estudantes universitários e suas relações com as autopercepções físicas, bem-estar subjectivo e felicidade ${ }^{1}$
}

\author{
Cláudia Dias \\ Nuno Corte-Real \\ Rui Corredeira \\ André Barreiros \\ Tânia Bastos \\ António Manuel Fonseca \\ Universidade do Porto - Portugal
}

\begin{abstract}
Resumo
A presente investigação analisou as relações entre a prática desportiva de estudantes universitários e o modo como estes se percepcionavam a si próprios e às suas vidas. Participaram nesta investigação 2284 estudantes de ambos os sexos, com idades entre os 18 e os 30 anos $(M=21,87 \pm 2,37)$, que preencheram um questionário sobre a sua prática desportiva, bem como versões portuguesas do Physical Self-Perception Profile (Fox \& Corbin, 1989), da Subjective Happiness Scale (Lyubomirsky \& Lepper, 1999), da Satisfaction With Life Scale (Diener, Emmons, Larsen, \& Griffin, 1985) e da Positive and Negative Affect Schedule (Watson, Clark, \& Tellegen, 1988). Em geral, a análise dos resultados revelou que os indivíduos que possuíam mais confiança física e que avaliavam a sua vida mais positivamente praticavam desporto mais regularmente; porém, verificou-se igualmente que quem se percebia menos forte fisicamente e tinha uma auto-estima mais baixa também praticava desporto mais regularmente do que os pares.
\end{abstract}

Palavras-chave: estudantes universitários; prática desportiva; autopercepções físicas; felicidade; bem-estar subjectivo

\begin{abstract}
University students'sport practice and its relations with physical self-perceptions, subjective well-being and happiness. The purpose of this study was to examine the relationships between university students' sport practice and the way they perceived themselves and their lives. Participated in this investigation 2284 male and female students, with ages between 18 and 30 years $(M=21.87 \pm 2.37)$, who filled out a questionnaire to assess their levels of sport practice, as well the Portuguese versions of the Physical Self-Perception Profile (Fox \& Corbin, 1989), the Subjective Happiness Scale (Lyubomirsky \& Lepper, 1999), the Satisfaction With Life Scale (Diener, Emmons, Larsen, \& Griffin, 1985) and the Positive and Negative Affect Schedule (Watson, Clark, \& Tellegen, 1988). Overall, the analysis of the results showed that students who were more physically confident and evaluated their lives more positively did sport more regularly; however, results also showed that students who reported lower levels of physical strength and self-esteem practiced sport more regularly than their peers.
\end{abstract}

Keywords: university students; sport practice; physical self-perceptions; happiness; subjective well-being.

$\mathrm{O}$ conceito de estilo de vida saudável não é ainda consensual entre os investigadores da área. De facto, enquanto os estudos mais clássicos se preocuparam com o consumo de álcool, consumo de tabaco, hábitos alimentares ou de actividade física, investigações mais recentes têm-se debruçado sobre comportamentos como o consumo de drogas e medicamentos, acidentes e comportamentos de risco, higiene dental, frequência de realização de exames médicos, actividades desenvolvidas nos tempos livres, comportamentos sexuais ou cuidados com aparência e higiene (Pastor, Balaguer, \& GarciaMerita, 1998).

No entanto, independentemente da interpretação que adoptemos, é inequívoco que a prática de actividade física regular tem um papel preponderante na promoção de um estilo de vida saudável (Balaguer \& Castillo, 2002), sendo cada vez mais forte a convicção, tanto por parte da população em geral como da comunidade científica, das vantagens físicas, psicológicas e sociais que lhe estão associadas em todas as idades. 
Nesse sentido, a promoção de hábitos de prática regular de actividade física deve constituir-se como um dos principais objectivos das políticas de educação para a saúde, principalmente se considerarmos que, apesar das evidências do papel da actividade física na qualidade de vida: (1) a maioria dos jovens não apresenta os níveis recomendados (Caspersen, Nixon, \& Durant, 1998); (2) a actividade física declina com a idade, registrando-se os declínios mais acentuados no fim da adolescência (entre os 15 e os 18 anos) e início da idade adulta (entre os 20 e os 25 anos) (Stephens, Jacobs, \& White, 1985); e (3) a adopção de comportamentos protectores de saúde na infância, adolescência e início da idade adulta pode determinar a qualidade da vida adulta e da velhice (Buckworth, 2001).

Promover a adopção por parte da população em geral, e dos jovens em particular, de práticas de actividade física regular e a sua manutenção ao longo do tempo pode todavia assumir-se como um grande desafio para as instituições e para os diferentes profissionais implicados neste processo. No caso específico dos estudantes universitários, tal desafio pode ser ainda maior. Com efeito, a nível internacional, dados do United States Department of Health and Human Services ([USDHHS], 1996) mostraram uma quebra no nível de actividade física no fim do ensino secundário e durante a universidade, do mesmo modo que uma investigação de Calfas, Sallis, Lovato e Campbell (1994) revelou que $47 \%$ dos estudantes norte-americanos diminuíam os níveis de actividade física quando entravam na universidade. Também em Espanha, investigações recentes vieram apontar no sentido da existência de um elevado sedentarismo por parte da comunidade universitária (Ruiz, García, \& Gómez, 2005; Sanz, 2005).

Parece portanto que os estudantes universitários, de uma forma geral, não estão preparados de forma eficaz para a transição de um sistema em que a realização de actividade física é obrigatória para um outro em que ela passa a ser voluntária (Sallis \& Mckenzie, 1991), exigindo uma responsabilidade individual que podem não possuir ou não estar dispostos a assumir. Por outro lado, para que se possa intervir na promoção de um estilo de vida activo e saudável neste contexto específico, importa compreender a natureza multifacetada desse estilo de vida, que ultrapassa largamente uma dimensão físico-comportamental, envolvendo também as dimensões social, cognitiva e afectiva.

Neste contexto, interessa salientar que a Psicologia Social e Motivacional contemporânea tem vindo a sublinhar a utilidade de que se reveste a abordagem sócio-cognitiva - em que a ênfase é colocada no papel das autopercepções - na compreensão dos sentimentos, expectativas e comportamentos dos indivíduos (Fiske \& Taylor, 1991). Ao nível do estudo das percepções que o indivíduo tem de si mesmo em diversos domínios (e.g., académico, social, físico), o autoconceito físico (i.e., o que sentimos e a importância que atribuímos à nossa aparência e competência física ou aptidão motora) tem vindo a ocupar uma posição única entre os diversos factores potencialmente determinantes da actividade física dos indivíduos, na medida em que define a identidade física e se constitui como a base da auto-estima e de muitos dos padrões de comportamentos neste e noutros contextos (Fox, 1997).

De facto, diversas investigações mostraram que um autoconceito positivo está ligado a uma maior atracção pela prática de actividade física (e.g., Brustad, 1988; Fox \& Corbin, 1989), e que há uma maior probabilidade das pessoas se envolverem em contextos de actividade física se se sentirem bem consigo próprias, confiantes e competentes naquilo que fazem (e.g., Marsh \& Craven, 1997; Marsh \& Perry, 2005). Mais especificamente, o indivíduo que possua autopercepções elevadas relativamente à sua competência física, em princípio, estará mais disponível para realizar actividade física, pois terá mais oportunidade de demonstrar competência. Por outro lado, pessoas com autopercepções físicas reduzidas provavelmente manter-se-ão afastadas da prática de actividade física e, consequentemente, da oportunidade de demonstrarem competência em determinada tarefa, resultando desta situação que a sua competência física percebida se manterá reduzida, constituindo um obstáculo ao início da actividade física (Harter, 1988; Lidwall, 2004). De forma inversa, um envolvimento regular em contextos de actividade física parece também possuir potencial para a mudança da forma como as pessoas se percebem fisicamente, levando-as a sentirem-se melhores consigo próprias, contribuindo para o seu bem-estar físico e mental e, em última instância, para a melhoria da sua qualidade de vida (Berger \& McInman, 1993; Buckworth \& Dishman, 2002; Fox, 1997, 1998).

Paralelamente, nos últimos anos tem vindo a crescer o interesse pela relação entre os estados afectivos e emocionais, entre os quais o bem-estar subjectivo e a felicidade, e a prática regular e sistemática de actividade física (e.g., Balaguer, 2000; Standage, Duda, \& Pensgaard, 2005). O bem-estar subjectivo consiste na forma como as pessoas avaliam a sua vida. Estas avaliações podem ser cognitivas (e.g., satisfação com a vida, sensação de realização pessoal) ou afectivas (a presença de emoções e estados de humor positivo e ausência de emoções e estados de humor negativos) (Diener, 2007; Diener \& Fujita, 1994; Diener \& Lucas, 2000). Nesse contexto, as pessoas experienciam bem-estar subjectivo quando estão envolvidas em actividades interessantes e se sentem satisfeitas com as respectivas vidas (bem-estar cognitivo), experienciando igualmente muito prazer e emoções agradáveis e pouco sofrimento e emoções desagradáveis (bem-estar afectivo) (Diener, 2000, 2007).

A existência de uma relação entre o bem-estar subjectivo e a prática de actividade física parece ser uma questão pacífica e aceita pelos investigadores da área (Biddle, 2000; Stephens, 1988). Por um lado, já foram encontradas associações claras entre a prática de actividade física e a satisfação com a vida (e.g., Balaguer, 2000). Por outro, os estados afectivos têm vindo a ser cada vez mais reconhecidos como um correlato fundamental da actividade física (Crocker \& Graham, 1995; Gauvin \& Rejesky, 1993), na medida em que diversas pessoas consideram o exercício físico uma estratégia eficaz para lidarem com estados de humor negativos (Erber, 1994, 1996; Thayer et al., 1994). A propósito, segundo Fox (1999), pelo menos 20 revisões de estudos já foram publicadas demonstrado a relação da prática de actividade física com estados afectivos positivos.

A felicidade pode ser entendida como uma avaliação positiva subjectiva da qualidade global da vida e reflecte o ajustamento global do indivíduo à vida (i.e., até que ponto a pessoa gosta de si e se sente bem com a vida que tem) (Cropanzano \& Wright, 
2001; Veenhoven, 2000). Além disso, na medida em que inclui a experiência de alegria, contentamento ou bem-estar positivo, combinada com a sensação de que a vida é boa, com significado, algo que "vale à pena", parece abranger componentes afectivos, mas também cognitivos (Lyubomirsky, 2001).

Importa salientar que embora seja muitas vezes apresentada como sinónimo de bem-estar subjectivo e de satisfação com a vida, Lyubomirsky e Lepper (1999) defendem que uma avaliação da felicidade subjectiva, na medida em que implica uma avaliação global, por parte de alguém, como uma pessoa feliz ou infeliz, reflecte uma categoria mais ampla do bem-estar e remete para um fenómeno psicológico mais global. Segundo aquelas autoras, a maior parte das pessoas é capaz de indicar em que medida se considera uma pessoa feliz (ou infeliz), não sendo esse juízo equivalente a uma simples soma dos seus níveis de afecto e de satisfação com a vida; um indivíduo pode avaliar-se como alguém muito feliz, apesar de a sua vida ser um apenas "um pouco feliz", e, de forma inversa, pode identificar-se, genericamente, como alguém infeliz, ainda que tenha experienciado diversos estados afectivos positivos num passado recente (e.g., o mês passado).

À semelhança das autopercepções físicas e do bem-estar subjectivo, a felicidade parece ter uma relação estreita com a actividade física. A título de exemplo, cabe destacar uma investigação recente de Tkach e Lyubomirsky (2006), em que, tendo sido estudadas as estratégias a que 500 estudantes universitários recorriam para aumentar ou manter a felicidade, se verificou que a prática de exercício e actividade física emergia como um forte preditor da felicidade.

É, pois, nesse contexto, e porque os dados actualmente disponíveis neste domínio do conhecimento, particularmente no que concerne à realidade portuguesa, não são ainda suficientemente esclarecedores, que interessa perceber melhor o padrão de inter-relações entre o envolvimento dos jovens universitários na actividade física e o modo como eles se percebem a si próprios fisicamente e avaliam a sua felicidade e o seu bem-estar. Considerando, no entanto, que a actividade física pode englobar actividades tão diversas como as actividades quotidianas (e.g., andar, tarefas domésticas), as actividades cíclicas (e.g., andar, correr, saltar à corda), os exercícios de preparação física (e.g., flexões de braços, estiramentos), ou a prática de desportos (Devis, 2000) - o que torna este conceito de difícil avaliação na sua totalidade (Corte-Real, 2006)-, optou-se por considerar nesta investigação apenas a prática desportiva (PD), entendida esta na linha da definição patrocinada pelo Conselho da Europa (1995), a qual estabelece que o desporto inclui todas as formas de actividade física que, por meio de uma participação organizada ou não, têm por objectivo a melhoria da condição física e psíquica, o desenvolvimento das relações sociais ou a obtenção de resultados em competições de todos os níveis. Ou seja, esta definição implica não só a intencionalidade do indivíduo para adoptar estes comportamentos, mas também um certo grau de regularidade, tornando o estudo da PD dos estudantes universitários uma questão relevante se o que pretendemos é desenvolver estratégias e encontrar soluções para facilitar um estilo de vida activo e saudável neste contexto específico.

\section{Método}

\section{Participantes}

A amostra foi constituída por 2284 estudantes, que frequentavam os diferentes anos de licenciatura, pós-graduação, mestrado e doutoramento (29,3\% do sexo masculino e $70,7 \%$ do sexo feminino), das diferentes faculdades (Arquitectura, Belas Artes, Ciências, Ciências da Nutrição e da Alimentação, Desporto, Direito, Economia, Engenharia, Farmácia, Ciências Biomédicas, Letras, Medicina, Medicina Dentária, Psicologia e Ciências da Educação) da Universidade do Porto (UP), a maior universidade portuguesa, com idades compreendidas entre os 18 e os 30 anos $(M=21,87 \pm 2,37)$.

\section{Instrumentos}

\section{Prática desportiva.}

No que respeita à PD efectuada pelos estudantes, foram avaliados indicadores relativos à frequência ( 1 a 3 vezes/ mês, 1 vez/mês, 2 a 3 vezes/semana, 4 a 5 vezes/semana, 6 a 7 vezes/semana) e duração habitual de cada sessão (menos de 20 minutos, entre 20 e 45 minutos, mais de 45 minutos) de treino das três modalidades mais praticadas. As respostas foram analisadas conjuntamente, tendo sido criados três grupos com níveis distintos de PD: (1) PD inexistente; (2) PD irregular (no máximo duas a três vezes/semana, menos de 20 minutos); e (3) PD regular (pelo menos duas a três vezes/semana, mais de 20 minutos/sessão).

\section{Autopercepções físicas}

Para avaliar as autopercepções físicas foi utilizado o PSPPp (Fonseca \& Fox, 2002), que é a versão portuguesa do Physical Self-Perception Profile (PSPP; Fox \& Corbin, 1989). Este instrumento é composto por 16 itens, divididos por quatro dimensões: confiança física (i.e., percepções acerca da capacidade desportiva e atlética para aprender técnicas desportivas e confiança em contextos desportivos de rendimento, mas também do nível de condição física e confiança em contextos de actividade física e fitness); força física (i.e., percepções acerca da força física, desenvolvimento muscular e confiança em situações que exigem força); aparência física (i.e., percepções acerca da atracção exercida nos outros pela sua figura ou físico, capacidade para manter um corpo atraente e confiança na aparência); e auto-estima física (i.e., sentimentos generalizados de alegria, satisfação, orgulho, respeito e confiança no "eu físico"). Relativamente a cada um dos itens, os inquiridos pronunciamse por meio de uma estrutura de resposta igual à proposta por Harter (1988), isto é, de "formato de estrutura alternativa”. Nesse formato, cada item consiste num par de afirmações contrárias (e.g., "algumas pessoas sentem que não são muito boas quando se trata de praticar desporto" mas "outras sentem que são realmente boas acerca de qualquer desporto"), devendo o inquirido, numa primeira fase, seleccionar a afirmação que lhe parece descrevêlo melhor, para, em seguida, indicar o grau de semelhança que entende que essa afirmação tem consigo (i.e., se entende que, no seu caso, a afirmação seleccionada é "realmente verdade", ou apenas "mais ou menos verdade"). Deste modo, é eliminada 
a tendência dos indivíduos responderem de acordo com o que consideram ser socialmente mais aceito ou desejável (Fox \& Corbin, 1989). Esta escala demonstrou anteriormente possuir boas propriedades psicométricas, nomeadamente no que respeita à sua estrutura factorial e consistência interna (cf., Fonseca \& Fox, 2002). Na presente investigação, o PSPP $p_{p}$ revelou também valores aceitáveis de consistência interna $\left(\alpha_{\text {confiança física }}=0,75\right.$; $\left.\alpha_{\text {força física }}=0,77 ; \alpha_{\text {aparência física }}=0,67 ; \alpha_{\text {auto-estima física }}=0,77\right)$.

\section{Felicidade.}

Para avaliar a felicidade foi utilizada a SHS $_{\mathrm{p}}$, versão traduzida e adaptada por Fonseca (2006a) da Subjective Happiness Scale (SHS; Lyubomirsky \& Lepper, 1999). A SHS é uma medida da felicidade subjectiva e global, composta por quatro itens respondidos numa escala tipo Likert de sete pontos e um resultado compósito único é obtido, calculando-se a média das respostas a esses itens. A SHS demonstrou possuir uma elevada consistência interna, estável em diferentes amostras ( $\alpha=$ 0,79 a 0,$94 ; M=0,86$ ), existindo também evidências de validade convergente e discriminante e de estabilidade temporal (cf., Lyubomirsky \& Lepper, 1999). Na versão utilizada no presente estudo, a escala de resposta variava entre 1 e 5, e não entre 1 e 7 , tendo sido igualmente encontradas evidências de uma elevada consistência interna $(\alpha=0,75)$.

\section{Bem-estar subjectivo.}

Para avaliar a satisfação com a vida foi utilizada a SWLS, versão traduzida e adaptada para a língua portuguesa por Fonseca (2006b) da Satisfaction With Life Scale (SWLS; Diener, Emmons, Larsen, \& Griffin, 1985). A SWLS foi desenvolvida para medir o componente cognitivo do bem-estar psicológico (i.e., a satisfação global com a vida) e é constituída por cinco itens respondidos numa escala tipo Likert que varia entre 1 (Nada satisfeito com a minha vida) e 7 (Muito satisfeito com a minha vida). A SWLS mostrou ter boas propriedades psicométricas, incluindo uma elevada consistência interna e boa validade convergente e discriminante (Pavot \& Diener, 1993). Na versão utilizada no presente estudo, a escala de resposta variava entre 1 e 5 , e não entre 1 e 7 como na escala original, tendo sido encontrada uma elevada consistência interna $(\alpha=0,80)$.

Para avaliar o afecto positivo e o afecto negativo foi utilizada a PANAS , versão traduzida e adaptada para a língua portuguesa por Fonseca (2006c) da Positive and Negative Affect Schedule (PANAS, Watson, Clark, \& Tellegen, 1988). Este instrumento é constituído por duas escalas com 10 itens cada, que visam avaliar duas dimensões conceptualmente independentes do estado de humor: afecto positivo (10 itens; e.g., “excitado”, “entusiasmado”, “inspirado”) e afecto negativo (10 itens; e.g., “angustiado”, “hostil”, “irritável”). Para o seu preenchimento, os participantes devem indicar em que medida experienciam as emoções listadas, recorrendo a uma escala tipo Likert de 5 pontos $(1=$ Nada; 5 = Muito $)$. Watson e colaboradores verificaram que as escalas possuem uma boa estrutura factorial e elevada consistência interna $\left(\alpha_{\text {afecto positivo }}=0,86-0,90 ; \alpha_{\text {afecto negativo }}=\right.$ $0,84-0,87)$. Na versão utilizada no presente estudo também foi evidente a existência de uma elevada consistência interna, quer para o afecto positivo $(\alpha=0,77)$, quer para o afecto negativo $(\alpha=0,82)$.

\section{Procedimentos}

Para a operacionalização do presente estudo, foram contactados os Conselhos Directivos das diferentes faculdades, por intermédio do Instituto de Recursos e Iniciativas Comuns da Universidade do Porto, os quais não só concederam a necessária autorização, como assumiram a responsabilidade pela distribuição e recolha dos questionários nas suas escolas. Cabe mencionar ainda que, para além da autorização institucional para a realização do estudo, foi igualmente solicitada a participação voluntária dos estudantes, aos quais foi assegurado o anonimato e confidencialidade dos dados recolhidos.

\section{Resultados}

Na Tabela 1, podem ser visualizados os dados relativos aos níveis de $\mathrm{PD}$ dos estudantes universitários envolvidos neste estudo. A análise destes níveis revelou que quase metade dos participantes não praticava qualquer tipo de modalidade desportiva; todavia, importa também salientar que aproximadamente 4 em cada 10 dos estudantes estavam envolvidos numa PD regular e frequente.

Tabela 1

Níveis de prática desportiva (PD) dos estudantes universitários; análise da amostra global.

\begin{tabular}{lrc}
\hline Níveis de prática desportiva & \multicolumn{1}{c}{$n$} & $\%$ \\
\hline PD inexistente & 1065 & 47,1 \\
PD reduzida & 300 & 13,3 \\
PD regular & 894 & 39,6 \\
\hline Total & 2259 & 100,0 \\
\hline
\end{tabular}

No que respeita às autopercepções físicas, os estudantes da Universidade do Porto revelaram níveis moderados, salientandose a autopercepção de força física (Tabela 2).

Tabela 2

Autopercepções físicas; análise da amostra global

\begin{tabular}{lc}
\hline \multicolumn{1}{c}{ Dimensões } & Média $\pm D P$ \\
\hline Confiança física & $2,45 \pm 0,71$ \\
Força física & $2,55 \pm 0,61$ \\
Aparência física & $2,45 \pm 0,47$ \\
Auto-estima física & $2,47 \pm 0,49$ \\
\hline
\end{tabular}

De forma semelhante, foram também evidentes níveis moderados de felicidade, satisfação com a vida e afecto positivo, o qual se sobrepôs claramente ao afecto negativo e ligeiramente à satisfação com a vida e à felicidade (Tabela 3).

Com o intuito de compreender a relação entre a prática de desporto e as variáveis psicológicas em estudo, procurámos inicialmente analisar diferenças nos níveis de PD entre os estudantes que apresentavam valores reduzidos e elevados 
ao nível das autopercepções físicas, felicidade e bem-estar subjectivo. Para a formação desses dois grupos considerou-se a média grupal de cada variável, à qual foi subtraído ou adicionado o respectivo valor de desvio-padrão. A partir dos valores obtidos, foram criados dois grupos, que foram comparados entre si: um grupo de estudantes cuja média individual os remetia para o extremo inferior da variável em questão, e um outro grupo cuja média individual os colocava no extremo superior.

Tabela 3

Felicidade e bem-estar subjectivo; análise da amostra global

\begin{tabular}{lc}
\hline \multicolumn{1}{c}{ Dimensões } & Média $\pm D P$ \\
\hline Felicidade geral & $3,51 \pm 0,61$ \\
Satisfação com a vida & $3,50 \pm 0,61$ \\
Afecto positivo & $3,55 \pm 0,47$ \\
Afecto negativo & $2,64 \pm 0,58$ \\
\hline
\end{tabular}

Assim, no que concerne às autopercepções físicas, foi evidente a existência de diferenças significativas entre a PD dos dois grupos, designadamente quando consideradas as dimensões de confiança física, força física e auto-estima física (Tabela 4).

Os estudantes com mais confiança nas suas capacidades desportivas e atléticas indicaram realizar uma PD mais regular do que os seus pares com percepções reduzidas a este nível. Com efeito, enquanto nos estudantes com percepções elevadas de confiança física $69 \%$ praticavam desporto de forma regular, existindo apenas 19\% de sedentários, nos estudantes com reduzida confiança física passava-se o inverso, na medida em que $65 \%$ não praticavam desporto e apenas $25 \%$ o faziam com regularidade.

Por outro lado, em relação às autopercepções de força e de auto-estima físicas parecia suceder o contrário. Nestes dois casos, para além de existir um maior sedentarismo por parte dos estudantes com autopercepções mais elevadas (63\% e 54\%, respectivamente) comparativamente aos estudantes com autopercepções mais reduzidas (28\% e 45\%, respectivamente), a percentagem de estudantes que praticava regularmente desporto era mais elevada entre os que tinham uma percepção mais negativa da sua força física (63\%) e se sentiam pior com o seu "eu físico" (45\%) do que nos sujeitos que se percebiam menos positivamente nestes domínios (26\% e 34\%, respectivamente).

A comparação dos níveis de PD dos estudantes em função dos seus níveis de felicidade e bem-estar subjectivo revelou também diferenças significativas em todas as variáveis envolvidas na análise (Tabela 5). De uma forma geral, os estudantes com níveis mais elevados de felicidade, satisfação

Tabela 4

Diferenças nos níveis de prática desportiva (PD) em função das autopercepções físicas

\begin{tabular}{|c|c|c|c|c|c|c|c|c|}
\hline \multirow[t]{2}{*}{ Níveis } & \multicolumn{2}{|c|}{$\begin{array}{c}\text { Confiança física } \\
(\%)\end{array}$} & \multicolumn{2}{|c|}{$\begin{array}{c}\text { Força física } \\
\text { (\%) }\end{array}$} & \multicolumn{2}{|c|}{$\begin{array}{c}\text { Aparência física } \\
(\%)\end{array}$} & \multicolumn{2}{|c|}{$\begin{array}{c}\text { Auto-estima física } \\
(\%)\end{array}$} \\
\hline & Baixa & Elevada & Baixa & Elevada & Baixa & Elevada & Baixa & Elevada \\
\hline PD inexistente & 65,1 & 19,1 & 28,2 & 63,3 & 48,1 & 48,8 & 45,0 & 54,3 \\
\hline PD reduzida & 9,9 & 12,3 & 9,2 & 11,1 & 10,4 & 15,2 & 10,1 & 11,7 \\
\hline \multirow[t]{2}{*}{ PD regular } & 25,0 & 68,7 & 62,6 & 25,7 & 41,5 & 36,0 & 45,0 & 34,0 \\
\hline & \multicolumn{2}{|c|}{$\begin{array}{c}\chi^{2}(2)=149,02 \\
p<0,001\end{array}$} & \multicolumn{2}{|c|}{$\begin{array}{c}\chi^{2}(2)=87,07 \\
p<0,001\end{array}$} & \multicolumn{2}{|c|}{$\begin{array}{c}\chi^{2}(2)=3,38 \\
p>0,05\end{array}$} & \multicolumn{2}{|c|}{$\begin{array}{c}\chi^{2}(2)=7,45 ; \\
p<0,05\end{array}$} \\
\hline
\end{tabular}

com a vida e afecto positivo, e mais reduzidos de afecto negativo revelaram-se fisicamente mais activos (com percentagens de estudantes sedentários a variarem entre os $40 \%$ e os $57 \%$ e de estudantes com PD regular a variarem entre os $47 \%$ e os $52 \%$ ) do que os seus colegas do grupo oposto (com percentagens de estudantes sedentários a variarem entre os $36 \%$ e os $40 \%$ e percentagens de estudantes com PD regular a variarem entre os $29 \%$ e os $36 \%$ ).

Uma outra forma de analisar a relação entre a PD e as variáveis relativas às autopercepções, felicidade e bem-estar subjectivo, passou pela análise das correlações existentes entre aquelas variáveis, a qual evidenciou a existência de correlações estatisticamente significativas em todos os casos (Tabela 6)

De facto, foi evidente que a PD se correlacionava positivamente com a dimensão da confiança física, das autopercepções físicas, a felicidade, a satisfação com a vida e o afecto positivo e negativamente com as dimensões da força física, da aparência física e da auto-estima física, das autopercepções físicas, e ainda com o afecto negativo. Convém mencionar, todavia, que, apesar da significância estatística de todas essas correlações, ao que não foi certamente alheia a dimensão da amostra, a sua magnitude, com excepção dos casos das dimensões da confiança e força físicas, das autopercepções físicas, foi inferior a 0,20. 
Tabela 5

Diferenças nos níveis de prática desportiva (PD) em função da felicidade e bem-estar subjectivo

\begin{tabular}{|c|c|c|c|c|c|c|c|c|}
\hline \multirow{2}{*}{ Níveis } & \multicolumn{2}{|c|}{$\begin{array}{c}\text { Felicidade geral } \\
(\%)\end{array}$} & \multicolumn{2}{|c|}{$\begin{array}{c}\text { Satisfação com } \\
\text { a vida (\%) }\end{array}$} & \multicolumn{2}{|c|}{$\begin{array}{l}\text { Afecto positivo } \\
\text { (\%) }\end{array}$} & \multicolumn{2}{|c|}{$\begin{array}{c}\text { Afecto negativo } \\
\text { (\%) }\end{array}$} \\
\hline & Baixa & Elevada & Baixa & Elevada & Baixa & Elevada & Baixa & Elevada \\
\hline PD inexistente & 54,6 & 37,0 & 56,4 & 40,2 & 56,2 & 36,5 & 36,4 & 57,1 \\
\hline PD reduzida & 9,6 & 11,4 & 12,1 & 13,2 & 15,1 & 11,8 & 16,6 & 10,9 \\
\hline \multirow[t]{2}{*}{ PD regular } & 35,8 & 51,6 & 31,5 & 46,6 & 28,7 & 51,7 & 47,0 & 32,0 \\
\hline & \multicolumn{2}{|c|}{$\begin{array}{c}\chi^{2}(2)=19,03 \\
p<0,001\end{array}$} & \multicolumn{2}{|c|}{$\begin{array}{c}\chi^{2}(2)=23,49 \\
p<0,001\end{array}$} & \multicolumn{2}{|c|}{$\begin{array}{c}\chi^{2}(2)=33,93 \\
p<0,001\end{array}$} & \multicolumn{2}{|c|}{$\begin{array}{c}\chi^{2}(2)=27,19 \\
p<0,001\end{array}$} \\
\hline
\end{tabular}

Tabela 6

Correlações entre a prática desportiva e autopercepções

físicas, felicidade e bem-estar subjectivo

\begin{tabular}{lrc}
\hline & $\begin{array}{c}\text { Prática } \\
\text { desportiva } \\
(r)\end{array}$ & $p$ \\
\hline Confiança física & 0,30 & $<0,001$ \\
Força física & $-0,26$ & $<0,05$ \\
Aparência física & $-0,05$ & $<0,05$ \\
Auto-estima física & $-0,10$ & $<0,001$ \\
Felicidade & 0,13 & $<0,001$ \\
Satisfação com a vida & 0,11 & $<0,001$ \\
Afecto positivo & 0,12 & $<0,001$ \\
Afecto negativo & $-0,12$ & $<0,001$ \\
\hline
\end{tabular}

\section{Discussão e conclusões}

A actividade física, de carácter recreativo ou inserida na prática de uma modalidade desportiva, tem um importante papel na promoção da saúde e na prevenção da doença, comportando, para além dos múltiplos benefícios no domínio físico, uma melhoria da saúde mental e qualidade de vida da pessoa. Por estas razões, a promoção de um estilo de vida activo nos jovens, especialmente nos jovens universitários constantemente tentados ou envolvidos em diversas actividades mais relacionadas com um estilo de vida sedentário, que acabam por se constituir como um bloqueio ou obstáculo à prática de actividade física - deve ser um objectivo prioritário.

Neste contexto, a presente investigação propunha-se analisar os níveis de PD e a sua relação com alguns factores intrapessoais que podem ser determinantes na adopção de um estilo de vida que inclua uma prática regular de desporto, nomeadamente as percepções que possuem relativamente a si próprios no domínio físico, bem como a avaliação que fazem da sua felicidade e bemestar, domínio em que a informação relativamente à população envolvida neste estudo é ainda escassa.

No que concerne aos resultados encontrados, um primeiro aspecto que importa realçar respeita ao elevado número de estudantes (praticamente metade da amostra) que não praticava qualquer tipo de modalidade desportiva. Este nível de sedentarismo, indesejável mas não surpreendente, está na linha de muitas investigações realizadas em populações de adolescentes, não só em Portugal (e.g., Corte-Real, 2006; Matos, Simões, Carvalhosa \& Canha, 2003; Santos, 2004), mas também no estrangeiro (e.g., Balaguer \& Castillo, 2002; Organização Mundial de Saúde, 2004). No que se refere mais especificamente à população universitária, existem também evidências de uma elevada taxa de inactividade física em jovens universitários, por exemplo espanhóis (e.g., Ruiz, Garcia, \& Gómez, 2005; Sanz, 2005) e norte-americanos (e.g., Calfas et al., 1994; USDHHS, 1996).

Da comparação dos índices de prática desportiva de estudantes com diferentes percepções relativamente a si mesmos e às suas vidas evidenciou-se, em primeiro lugar, que os estudantes que confiavam mais nas suas capacidades desportivas e atléticas praticavam desporto mais regularmente do que os que tinham percepções reduzidas neste domínio. Todavia, foi igualmente evidente que os estudantes que revelaram autopercepções mais reduzidas de força física e desenvolvimento 
muscular, bem como os que tinham sentimentos mais negativos relativamente ao seu "eu físico" (i.e., que tinham uma autoestima física mais reduzida), praticavam desporto de forma mais regular do que os seus colegas com autopercepções mais elevadas nestes domínios.

Complementarmente, foi possível verificar que, em termos globais, a PD se encontrava positivamente associada à autopercepção da confiança física e negativamente à autopercepção da força física, tendo ainda sido identificada uma relação negativa, embora de reduzida magnitude, entre a PD e a autopercepção da aparência física e a auto-estima física. De uma forma genérica, e com excepção do caso da autopercepção da confiança física, estes resultados não são consonantes com a assunção do autoconceito como uma variável que facilita e promove comportamentos de actividade física e adesão à PD (Fox \& Corbin, 1989; Horn \& Amorose, 1998; Marsh, 1997), nem com investigações realizadas nesta área que destacam a existência de uma relação entre autopercepções físicas positivas e a prática de desporto e exercício (e.g., Marsh \& Craven, 1997; Marsh \& Perry, 2005).

No entanto, Biddle (1997) alertou para que em muitos casos - independentemente do reportado pela generalidade das investigações, relativamente à relação estreita existente entre a forma como as pessoas se percebem e o seu envolvimento em actividades físicas e desportivas - as pessoas, mesmo que não se percebam como competentes relativamente a uma determinada actividade, decidem ainda assim realizá-la. Além disso, no que se refere concretamente à auto-estima, embora haja uma assunção generalizada de que a melhoria da auto-estima global constitui um resultado automático da participação na actividade física (Spence, McGannon \& Poon, 2005), estudos recentes (e.g., Calfas \& Taylor, 1994; McAuley, Blissmer, Katula, Duncan, \& Mihalko, 2000) questionaram a magnitude da mudança que pode ser esperada na auto-estima global como resultado da participação na actividade física, fornecendo assim algum enquadramento para a praticamente inexistente relação encontrada no presente estudo entre estas variáveis.

Suplementarmente, importará realçar que é perfeitamente admissível que sejam os jovens universitários com autopercepções físicas mais reduzidas a decidirem investir mais tempo e esforço a praticar desporto precisamente devido à sua intenção de melhorar a sua força e aparência físicas. Na verdade, isso mesmo foi verificado numa investigação realizada por Fonseca, Lago e Mota (1997), com praticantes de ginástica de academia, na qual foi evidente que as participantes que avaliavam a sua aparência física menos positivamente e que menos bem se sentiam com a sua componente física eram as mais assíduas às aulas. Ainda assim, importará recordar, uma vez mais, que a magnitude da correlação da aparência física com a PD era muito reduzida, praticamente inexistente.

Quanto à relação negativa identificada entre a autopercepção da força física e a PD dos estudantes, importará não esquecer que, muitas vezes, são exactamente os indivíduos com excesso de peso - que geralmente não praticam qualquer tipo de desporto - que têm igualmente os níveis mais elevados de força física, circunstância que, podendo ter contribuído, pelo menos parcialmente, para os resultados encontrados neste estudo deverá, em nossa opinião, ser tida em consideração em estudos futuros. Os resultados sugeriram igualmente a existência de uma relação estreita entre a regularidade da PD e a felicidade e o bem-estar subjectivo. Com efeito, os jovens que exibiam níveis mais elevados de felicidade e de bem-estar praticavam desporto com maior regularidade do que aqueles que se sentiam menos felizes e que experienciavam menos bem-estar; no mesmo sentido, as análises correlacionais destacaram a existência de associações positivas entre as referidas variáveis.

Estes dados vão parcialmente ao encontro dos resultados de uma investigação de Balaguer (2000), com adolescentes espanhóis, e de um estudo de Esperança (2005), com jovens portugueses com necessidades educativas especiais, que permitiram identificar uma relação entre a PD regular e níveis mais elevados de satisfação com a vida. Adicionalmente, são também convergentes com investigações que demonstraram relações consistentes entre a prática de desporto e actividade física e estados afectivos mais positivos (e.g., Crocker \& Graham, 1995; Gauvin \& Rejesky, 1993).

No que respeita à relação entre a $\mathrm{PD}$ e a felicidade e o bem-estar subjectivo, uma questão que, não tendo sido abordada na presente investigação, deverá, em nossa opinião, merecer a atenção futura dos investigadores, até pelo modo como poderá condicionar a intervenção neste contexto, prende-se com a determinação da sua direccionalidade. Será a PD mais regular que promove níveis mais elevados de felicidade e de bem-estar subjectivo (o que será extremamente positivo, considerando que estas sensações se estendem a vários domínios da vida da pessoa, comportando múltiplos benefícios no que respeita à melhoria da qualidade de vida global dos estudantes), ou, pelo contrário, será que são as pessoas que avaliam globalmente a sua vida de forma mais positiva que estão mais disponíveis, em termos emocionais e psicológicos, para se envolverem e manterem uma PD sistemática?

De resto, somos de opinião que a mesma questão poderá e deverá ser colocada relativamente à relação entre a PD e as autopercepções físicas, principalmente se se atender à relação negativa encontrada na presente investigação entre algumas das suas dimensões e a PD. De facto, será distinto concluir que são as autopercepções físicas mais reduzidas dos indivíduos que os levam a envolverem-se mais activamente em contextos desportivos, com o intuito de melhorar a sua componente física, ou, por outro lado, descobrir que uma PD regular promove autopercepções físicas mais reduzidas, a partir, por exemplo, do confronto com indivíduos com características físicas mais elevadas.

A propósito desta questão, consideramos ser importante destacar que, ainda que relativamente ao autoconceito e desempenho, Marsh e Perry (2005) defendem um modelo de efeitos recíprocos, no qual o autoconceito e o desempenho estão reciprocamente relacionados e são mutuamente reforçadores, de forma que cada um deles será negativamente afectado se o outro for ignorado. Futuramente, poderá ser interessante abordar este modelo de forma mais aprofundada, não só em relação às autopercepções físicas, mas também no que respeita à felicidade e ao bem-estar subjectivo, realizando estudos que recorram a desenhos metodológicos que permitam inferências sobre a 
causalidade das relações.

Adicionalmente, futuras investigações poderão também procurar explicar, por exemplo, o papel de outras variáveis moderadoras (e.g., idade, sexo) ou mediadoras (e.g., autoeficácia) na relação entre a PD, a felicidade e o bem-estar subjectivo, nomeadamente na população universitária. Como referimos anteriormente, esta é uma população muito específica, com necessidades muito particulares e, como tal, não se podem generalizar resultados obtidos com amostras de outras populações ou grupos etários a esta população.

Entretanto, a presente investigação, ao permitir identificar a relação existente entre a forma como os estudantes universitários que participaram neste se percebiam a si mesmo e às suas vidas e a sua PD, poderá constituir-se como uma "chamada de atenção” para a importância destas variáveis psicológicas serem consideradas como objectivos prioritários em intervenções que visem resultados positivos ao nível da saúde física e mental, não só nos jovens universitários, mas também na população em geral.

\section{Referências}

Balaguer, I. (2000). Un estudio sobre los predictores de los estilos de vida saludables de los adolescentes valencianos. Valência: Dirección General de la Salut Pública, Conselleria de Sanitat.

Balaguer, I., \& Castillo, I. (2002). Actividad física, ejercicio físico y deporte en la adolescencia temprana. In I. Balaguer (Org.), Estilos de vida en la adolescencia (pp. 37-63). Valencia: Promolibro

Berger, B. G., \& McInman, A. (1993). Exercise and the quality of life. In R. N. Singer, M. Murphey, \& L.K. Tennant (Orgs.), Handbook of research on sport psychology (pp. 729-760). Nova York, NY: Macmillan.

Biddle, S. J. H. (1997). Cognitive theories of motivation and the self. In K. R. Fox (Org.), The physical self: From motivation to well-being (pp. 59-82). Champaign, IL: Human Kinetics.

Biddle, S. J. H. (2000). Emotion, mood and physical activity. In S. J. H.Biddle, K. R. Fox, \& S. H. Boutcher (Orgs.) Physical activity and psychological well-being (pp. 63-87). Londres: Routledge.

Brustad, R. (1988). Affective outcomes in competitive youth sport: The influence of intrapersonal and socialization factors. Journal of Sport \& Exercise Psychology, 10, 307-321.

Buckworth, J. (2001). Exercise adherence in college students: Issues and preliminary results. Quest, 53, 335-345.

Buckworth, J., \& Dishman, R. (2002). Self-esteem. In J. Buckworth \& R. Dishman (Orgs.), Exercise Psychology (pp. 155-175). Champaign, Illinois: Human Kinetics.

Calfas, K. J., Sallis, J. F., Lovato, C. Y., \& Campbell, J. (1994). Physical activity and its determinants before and after college graduation. Medicine, Exercise, Nutrition and Health, 3, 323-334.

Calfas, K. J., \& Taylor, W. C. (1994). Effects of physical activity on psychological variables in adolescents. Pediatric Exercise Science, 6, 406-423.

Caspersen, C. Nixon, P. \& Durant, R. (1998). Physical activity epidemiology applied to children and adolescents. Exercise and Sport Sciences Reviews, 26, 341-403

Conselho da Europa (1995). The significance of sport for society - Health, socialization, economy: A scientific review. Strasbourg: Council of Europe Press.

Corte-Real, N. (2005). Desporto, saúde e estilos de vida: diferentes olhares, objectivos e subjectivos, sobre os comportamentos dos adolescentes. Dissertação de doutoramento não-publicada, Faculdade de Desporto da Universidade do Porto, Porto.
Crocker, P. R. E., \& Graham, T. R. (1995). Emotion in sport and physical activity: the importance of perceived individual goals. International Journal of Sport Psychology, 26, 117-137.

Cropanzano, R., \& Wright, T. A. (2001). When a "happy" worker is really a "productive" worker: a review and further refinement of the happy productive worker thesis. Consulting Psychology Journal: Practice and Research, 53, 182-197.

Devis, J. (2000). Actividad física, deporte y salud. Barcelona: Inde.

Diener, E. (2000). Subjective well-being: the science of happiness and a proposal for a national index. American Psychologist, 55(1), 34-43.

Diener, E. (2007). Frequently Asked Questions (FAQ's) about subjective wellbeing (happiness and life satisfaction): a primer for reporters and newcomers. Recuperado de http://www.psych.uiuc.edu/ ediener/faq.html, em 23 de março de 2007

Diener, E., Emmons, R. A., Larsen, R. J., \& Griffin, S. (1985). Satisfaction with Life Scale. Journal of Personality Assessment, 49(1), 71-75.

Diener, E., \& Fujita, F. (1994). Social comparisons and subjective well-being. In P. Buunk \& F. X. Gibbons (Orgs.), Health, coping, and well-being (pp. 329-357). Mahwah, New Jersey: Lawrence Erlbaum.

Diener, E., \& Lucas, R. E. (2000). Subjective emotional well-being. In M. Lewis \& J. M. Haviland (Orgs.), Handbook of emotions ( $2^{\underline{a}}$ ed., pp. 325-337). Nova York: Guilford.

Erber, R. (1996). The self-regulation of moods. In L. L. Martin \& A. Tesser (Orgs.), Striving and feeling: interactions among goals, affect, and self-regulation (pp. 251-275). Hillsdale, New Jersey: Lawrence Erlbaum.

Esperança, J. (2005). Estilos de vida, actividade física e satisfação com a vida: um estudo exploratório realizado com jovens com necessidade educativas especiais de várias escolas do país. Dissertação de mestrado não-publicada, Faculdade de Ciências do Desporto e de Educação Física, Porto.

Fiske, S. T., \& Taylor, S. E. (1991). Social cognition. Nova York: McGrawHill.

Fonseca, A. M. (2006a). Versão portuguesa da Subjective Happiness Scale: A Escala da Felicidade Subjectiva (SHS ). Manuscrito não-publicado, Faculdade de Desporto, Universidade do Porto, Porto.

Fonseca, A. M. (2006b). Versão portuguesa da Satisfaction With Life Scale: A Escala da Satisfação com a Vida $\left(S W L S_{\mathrm{p}}\right)$. Manuscrito não-publicado, Faculdade de Desporto, Universidade do Porto, Porto.

Fonseca, A. M. (2006c). Versão portuguesa da Positive and Negative Affect Schedule: A Escala dos Afectos Positivos e Negativos (PANAS ${ }_{\mathrm{p}}$ ). Manuscrito não-publicado, Faculdade de Desporto, Universidade do Porto, Porto.

Fonseca, A. M., \& Fox, K. R. (2002). Como avaliar o modo como as pessoas se percebem fisicamente? Um olhar sobre a versão portuguesa do Physical Self-Perception Profile (PSPP). Revista Portuguesa de Ciências do Desporto, 2(5), 11-23.

Fonseca, A. M., Lago, J., \& Mota, J. (1997). Estudo inicial sobre a assiduidade e as autopercepções físicas e de coesão de grupo em classes de ginástica de academia. Actas da V Conferência Internacional Avaliação Psicológica: Formas e contextos \& III Mostra de Provas Psicológicas em Portugal (pp. 450-460). Braga: APPORT \& Universidade do Minho.

Fox, K. R. (1997). The physical self and processes in self-esteem development. In K. R. Fox (Org.), The physical self: from motivation to well-being (pp. 111-139). Champaign, Illinois: Human Kinetics.

Fox, K. R. (1998). Advances in the measurement of the physical self. In J. Duda (Org.), Advances in Sport and Exercise Psychology Measurement (pp. 295310). Morgantown, West Virginia: Fitness Information Technology.

Fox, K. R. (1999). The influence of physical activity on mental well-being. Public Health Nutrition, 2(3a), 411-418.

Fox, K. R., \& Corbin, C. B. (1989). The Physical Self-Perception Profile: Development and preliminary validation. Journal of Sport \& Exercise Psychology, 11, 408-430.

Gauvin, L., \& Rejeski, W. J. (1993). The exercise-induced feeling inventory: Development and initial validation. Journal of Sport and Exercise 
Psychology, 15, 403-423.

Harter, S. (1988). The self-perception profile for adolescents. Denver, Colorado: Denver University.

Horn, T., \& Amorose, A. (1998). Sources of competence information. In J. L. Duda (Org.), Advances in sport and exercise psychology measurement (pp. 49-63). Morgantown, West Virginia: Fitness Information Technology.

Lidwall, M. (2004). Exercising the self: On the role of exercise, gender and culture in physical self-perceptions. Recuperado em 01 de setembro de 2004, de http://urn.kb.se/resolve?urn=um:nbn:se:diva-187>ISBN91-7265-871-1.

Lyubomirsky, S. (2001). Why are some people happier than others? The role of cognitive and motivational processes in well-being. American Psychologist, 56, 3, 239-249.

Lyubomirsky, S., \& Lepper, H. (1999). A measure of subjective happiness: Preliminary reliability and construct validation. Social Indicators Research, 46(2), 137-155.

McAuley, E., Blissmer, B., Katula, J., Duncan, T. E., \& Mihalko, S. L. (2000). Physical activity, self-esteem, and self-efficacy relationships in older adults: a randomized controlled trial. Annals of Behavioral Medicine, 22, 131-139.

Marsh, H. W. (1997). The measurement of physical self-concept: a construct validation approach. In K. Fox (Org.), The physical self: from motivation to well-being (pp. 27-58). Champaign, Illinois: Human Kinetics.

Marsh, H. W., \& Craven, R. (1997). Academic self-concept: beyond the dustbowl. In G. D. Phye (Org.), Handbook of classroom assessment (pp. 131-198). San Diego, California: Academic.

Marsh, H. W., \& Perry, C. (2005). Self-concept contributes to winning gold medals: Causal ordering of self-concept and elite swimming performance. Journal of Sport \& Exercise Psychology, 27, 71-91.

Matos, M., Simões, C., Carvalhosa, S., \& Canha, L. (2003). Saúde e estilos de vida em jovens portugueses: Estudo nacional da rede europeia HBSC/ OMS (1996). Lisboa: Faculdade de Motricidade Humana, Universidade Técnica de Lisboa.

Organização Mundial de Saúde (2004). Young people’s health in context: Health behaviour in school-aged children (HBSC) study. International Report from the 2001/ 2002 Survey. Copenhagen: Autor.

Pastor, Y., Balaguer, I., \& Garcia-Merita, M. (1998). Una revisión sobre las variables de estilos de vida saludables. Revista de Psicologia de la Salud, 10(1), 15-52.

Pavot, W., \& Diener, E. (1993). Review of the Satisfaction With Life Scale. Psychological Assessment, 5(2), 164-172.

Ruiz, F., Garcia, M. E. \& Gómez, M. (2005). Práctica de actividad físico- deportiva de tiempo libre. In F. Ruiz, M. E. Garcia \& M. Gomez (Orgs.), Hábitos físico-deportivos en centros escolares y universitarios (pp. 137-162). Madrid: Gymnos.

Sallis, J. F., \& Mckenzie, T. L. (1991). Physical education's role in public health. Research Quarterly for Exercise and Sport, 62, 124-137.

Santos, M. P. (2004). Factores de influência da actividade fisica em adolescentes: estudo da influência da posição sócio-económica, comportamentos sedentários e características do ambiente. Dissertação de doutoramento não-publicada, Faculdade de Ciências do Desporto e de Educação Física da Universidade do Porto, Porto.

Sanz, E. (2005). La práctica físico-deportiva de tiempo libre en universitarios: análisis y propuestas de mejora. Logroño: Universidade de La Rioja, Servicio de Publicaciones.

Spence, J. C., McGannon, K. R., \& Poon, P. (2005). The effect of exercise on global self-esteem: a quantitative review. Journal of Sport \& Exercise Psychology, 27, 311-334.

Standage M., Duda, J. L., \& Pensgaard, A. M. (2005). The effect of competitive outcome and task-involving, ego-involving, and cooperative structures on the psychological well-being of individuals engaged in a co-ordination task: a self-determination approach. Motivation and Emotion, 29(1), 41-68.

Stephens, T. (1988). Physical activity and mental health in the United States and Canada: evidence from four population surveys. Preventive Medicine, 17, 35-47.

Stephens, T., Jacobs, D. R., \& White, C. C. (1985). A descriptive epidemiology of leisure-time physical activity. Public Health Reports, 100, 147-158.

Thayer, R. E., Newman, R. \& McClain T. M. (1994). Self-regulation of mood: strategies for changing a bad mood, raising energy, and reducing tension. Journal of Personality and Social Psychology, 67, 910-925.

Tkach, C., \& Lyubormisky, S. (2006). How do people pursue happiness? Relating personality, happiness-increasing strategies and well-being. Journal of Happiness Studies, 7, 183-225.

United States Department of Health and Human Services. (1996). Physical activity and health: a report of the Surgeon General. Atlanta: Autor.

Veenhoven, R. (2000). Freedom and happiness: a comparative study in fortyfour nations in the early 1990s. In E. Diener \& E. M. Suh (Orgs.), Culture and subjective well-being (pp. 257-288). Cambridge, Massachusetts: The MIT Press.

Watson, D., Clark, L. A., \& Tellegen, A. (1988). Development and validation of brief measures of positive and negative affect: The PANAS scales. Journal of Personality and Social Psychology, 54(6), 1063-1070. 
Cláudia Salomé Lima Dias, doutora em Psicologia do Desporto pelo Instituto de Educação e Psicologia, Universidade do Minho, Portugal, é Professora Auxiliar na Faculdade de Desporto, Universidade do Porto, Portugal. Endereço para correspondência: Gabinete de Psicologia do Desporto; Faculdade de Desporto; Rua Dr. Plácido Costa, 91; 4200-450 Porto; Portugal. Telefone: +351 225074 700. Fax: +351 225500689. E-mail: cdias@fade.up.pt

Nuno José Corte-Real Correia Alves, doutor em Ciência do Desporto pela Faculdade de Desporto, Universidade do Porto, Portugal, é Professor Auxiliar na Faculdade de Desporto da mesma instituição. E-mail: ncortereal@fade.up.pt

Rui Manuel Nunes Corredeira, doutor em Ciência do Desporto pela Faculdade de Desporto, Universidade do Porto, Portugal, é Professor Auxiliar na Faculdade de Desporto da mesma instituição. E-mail: rcorredeira@ fade.up.pt

André Neves Barreiros, mestre em Psicologia do Desporto pela Faculdade de Motricidade Humana, Universidade Técnica de Lisboa, Portugal, é bolsista de doutorado em Ciência do Desporto, Fundação para a Ciência e Tecnologia, Portugal. E-mail: abarreiros@fade.up.pt

Tânia Cristina Lima Bastos, licenciada em Desporto e Educação Física pela Faculdade de Desporto, Universidade do Porto, Portugal, é bolsista de doutorado em Ciência do Desporto, Fundação para a Ciência e Tecnologia, Portugal. E-mail: tbastos@fade.up.pt António Manuel Leal Ferreira Mendonça da Fonseca, doutor em Ciência do Desporto e Agregação em Psicologia do Desporto, Universidade do Porto, Portugal, é professor catedrático na Faculdade de Desporto da mesma instituição. E-mail: afonseca@fade.up.pt 